\title{
Band gaps from ring resonators and structural periodicity
}

\author{
Yabing Qi, Bo Hou and Weijia Wen ${ }^{1}$ \\ Department of Physics, The Hong Kong University of Science and Technology, \\ Clear Water Bay, Kowloon, Hong Kong, People's Republic of China \\ E-mail: phwen@ust.hk
}

Received 4 October 2004, in final form 17 November 2004

Published 3 February 2005

Online at stacks.iop.org/JPhysD/38/590

\begin{abstract}
The electromagnetic band gap properties through a two-dimensional plate constructed with centimetre-sized spherical foam balls surrounded by aluminium rings are studied in the microwave range of $0.7-18 \mathrm{GHz}$. We observe from both experiments and finite-difference time-domain simulations that multiple band gaps appear and correspond to different resonant modes localized in the rings. The principal stop band, the lowest mode, appears at low frequencies and is robust to any lattice structure, even disorder. However, the remaining auxiliary stop bands, caused by high-order resonances, are usually located at higher frequencies and are more sensitive to the configuration of the structure formed by the ring resonators. We note that the stop bands would be broadened and strongly attenuated if more layers of the same plates were packed together. In addition, even or odd modes can be excited under different orientations of the rings with respect to the polarization of the illuminating radiation.
\end{abstract}

\section{Introduction}

Photonic crystals (PCs) have stimulated increasing interest due to their wide application in optical communications at frequencies ranging from the visible to the microwave [1-4]. Both two- and three-dimensional PCs have been developed and studied extensively in the past decades [5-9]. Compared with the two-dimensional PC structure, the three-dimensional PC structure has the advantage of having stop bands in a broader range of incident angles; however, realization of a three-dimensional PC structure is extremely difficult on the scale of the visible frequency regime. The conventional microlithography approach is required, which is complex and time-consuming. It has been found that the inverse-opal technique and the glancing angle deposition (GLAD) method are suitable for microfabrication of three-dimensional PCs in the optical or infrared frequencies [10-12]. Recently, the introduction of a metal scattering centre with a small volume fraction as the composite material instead of pure dielectrics has allowed the construction of so-called metallodielectric photonic crystals (MDPCs) [13-15]. This class of composite

1 Author to whom any correspondence should be addressed.
PC exhibits much larger stop bands and deeper band rejection than the conventional PC made of only dielectrics. Very recently, left-handed materials (LHMs) made of conducting elements have attracted a lot of attention because of their peculiar property of displaying simultaneously negative permittivity, $\varepsilon$, and negative permeability, $\mu$, [16-19]. Although most photonic band gap (PBG) materials are commonly composed of dielectric or metal scatterers or both arranged in a periodic way, the aperiodic arrangement of these building blocks has been observed to exhibit frequency forbidden regions [20,21].

Split ring resonators (SRRs) are extensively used to build LHMs, providing the obtainment of negative $\mu$ through magnetic coupling resonance $[17,18]$. However, electric field induced resonance is also found to exist in SRRs when electromagnetic (EM) waves illuminate the SRR with the $E$ vector parallel to the gap-bearing sides [22,23], for which the result was limited to the low-frequency regime. The resonant current distribution at the stop band frequency was shown to be a circular current with its maximum amplitude at the side opposite to the gap. Actually the distribution is the first harmonic mode of the resonant current, i.e. the lowest resonant mode, and should certainly be responsible for the 
lowest frequency band gap [24]. In this study, we investigated the band gap properties of the resonator composed of one split ring in the frequency range spanning from 0.7 to $18 \mathrm{GHz}$. We found that through experiments and finite-difference timedomain (FDTD) simulations, multiple band gaps can be obtained. We also found that the lowest gap, or the principal stop band, is very robust to any lattice structure, while higher frequency gaps or auxiliary stop bands are sensitive to its neighbours. There is an analogous effect occurring in MDPCs, where the lowest gap, which originates from the dipole particle-plasmon modes, is also quite structure-insensitive and survives even in the presence of disorder [25]. Here we showed that the underlying reason is that the ring is excited into different resonant modes.

\section{Samples and measurements}

In the construction of our samples, a piece of paper with lateral dimensions of $35 \mathrm{~cm} \times 35 \mathrm{~cm}$ was used as the substrate. The building block was a single ring resonator: a foam ball, $25 \mathrm{~mm}$ in diameter, was closely surrounded by an aluminium ring (width $2 \mathrm{~mm}$ and thickness $0.1 \mathrm{~mm}$ ) with an opening gap of $1 \mathrm{~mm}$ (see figure $1(b)$ inset for details of the geometry). The building blocks were affixed to the paper substrate to construct the different planar structures shown in figure 1, with the centre-to-centre separation between the neighbouring balls being $28 \mathrm{~mm}$ except in the random case. Two identical doubleridged waveguide horn antennae (RHODE \& SCHWARZ HF906) were employed as the microwave generator and receiver, which were $68 \mathrm{~cm}$ apart from each other. An S-parameter network analyser (HP Agilent 8720ES) was connected to the horns to measure the transmission spectrum in the range of $0.7-18 \mathrm{GHz}$. The ball-array structures were placed on top of a rotary stage, $15 \mathrm{~cm}$ from the receiving horn.

\section{Results and discussions}

The transmission at normal incidence of TEM waves for samples with different configurations of the ball arrangements was measured and the results are plotted as open circles in figures $1(a)-(e)$, where the data are normalized with respect to the free space spectrum. The plate constructed with only foam balls was used as the control sample. It can be seen from figure 1 $(a)$ that the fluctuation for the control sample was within $\pm 1 \mathrm{~dB}$, which indicates that both the paper substrate and the foam balls are totally transparent in our testing range. Figure 1(b) shows the transmittance of EM waves for the ball arrangement with a square lattice. In order to get a clear picture of the stop band position, the band edge is identified as the frequencies where the transmittance value is $-5 \mathrm{~dB}$ and, consequently, the middle frequency of a stop band can be defined as $f_{c}=\left(f_{-}+f_{+}\right) / 2$, where $f_{-}$and $f_{+}$are frequencies for the lower edge and the upper edge, respectively. Four stop bands can be identified and are marked as $\mathrm{A}, \mathrm{B}, \mathrm{C}$ and $\mathrm{D}$, with their middle frequencies located at $1.57 \mathrm{GHz}, 5.57 \mathrm{GHz}, 9.41 \mathrm{GHz}$ and $11.2 \mathrm{GHz}$, respectively. The maximum rejection at these stop bands decreases when the frequency increases. All such salient features can be detected in the structures with hexagonal (figure $1(c)$ ) and quasicrystal (figure $1(d)$ ) patterns. We note that the same

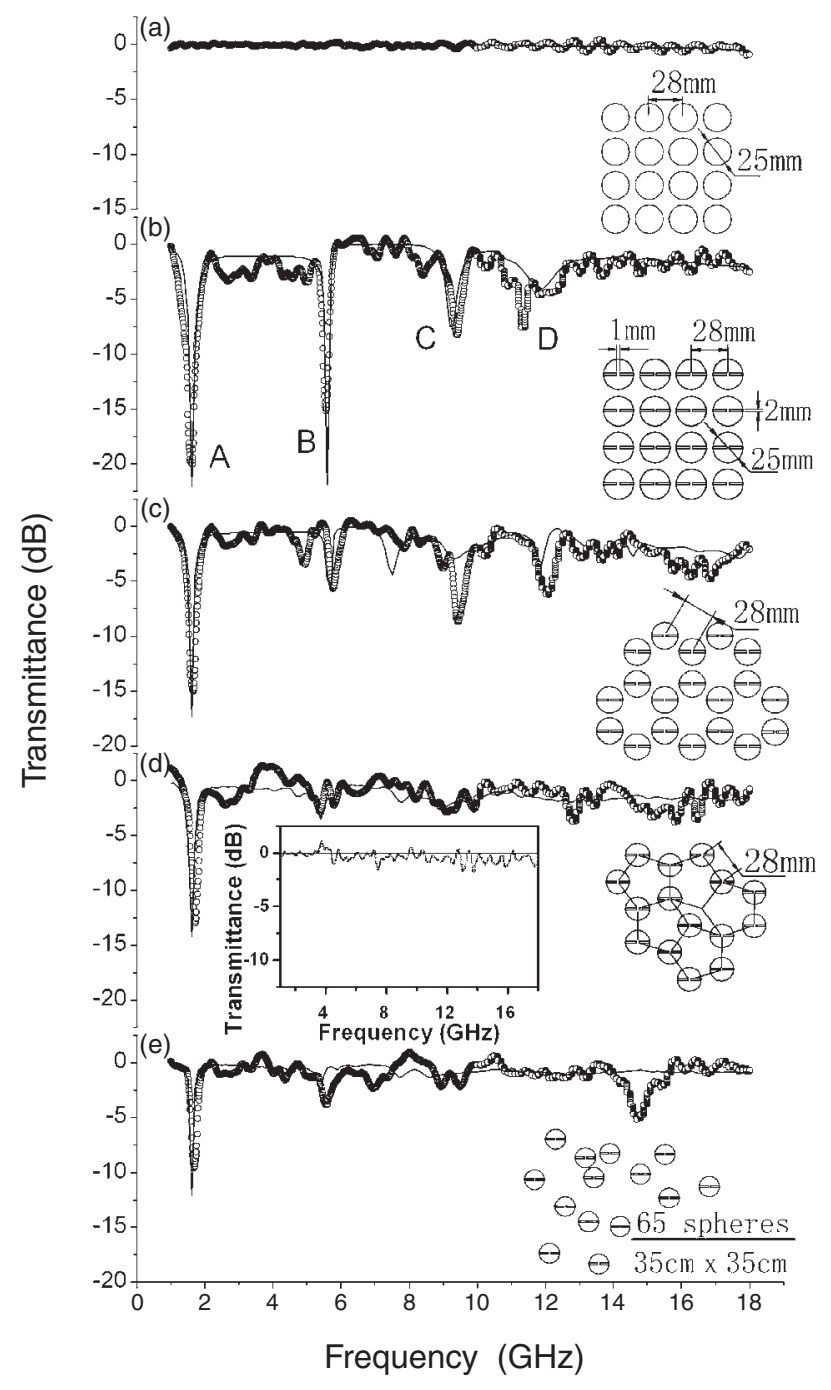

Figure 1. The transmittance at normal incidence of TEM waves for $(a)$ a control sample, $(b)$ a square lattice structure, $(c)$ a hexagonal lattice structure, $(d)$ a quasicrystal-structure and $(e)$ a random configuration, with $E$ parallel to the ring plane and $H$ perpendicular to it. The inset of $(d)$ shows the normal transmittance of the quasicrystal structure when $H$ is parallel to the ring plane and $E$ is perpendicular to it. The open circles represent the experimental measurements and the solid lines represent the simulations.

phenomena could be observed for the structure where the balls were randomly distributed (see figure 1(e)). One thing that should be emphasized here is that these band gaps were measured when the TEM mode microwave was normally incident on the samples with the magnetic field perpendicular to the ring plane. However, there exists no band essentially in any structure if the incident magnetic field is parallel to the ring plane (see figure $1(d)$ inset for the quasicrystal-structure case) since there is neither electric nor magnetic coupling of EM waves to the ring resonators at this polarization. In figure 1 the principal stop band has almost the same middle frequency, $\sim 1.7 \mathrm{GHz}$, regardless of the configuration, which indicates that such a stop band is mainly dictated by the characteristics of the building block, i.e. the individual ball. In sharp contrast to the principal stop band, all the auxiliary stop bands are more sensitive to the configuration. In other words, the auxiliary 


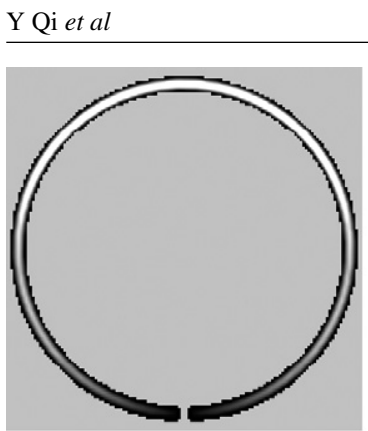

A

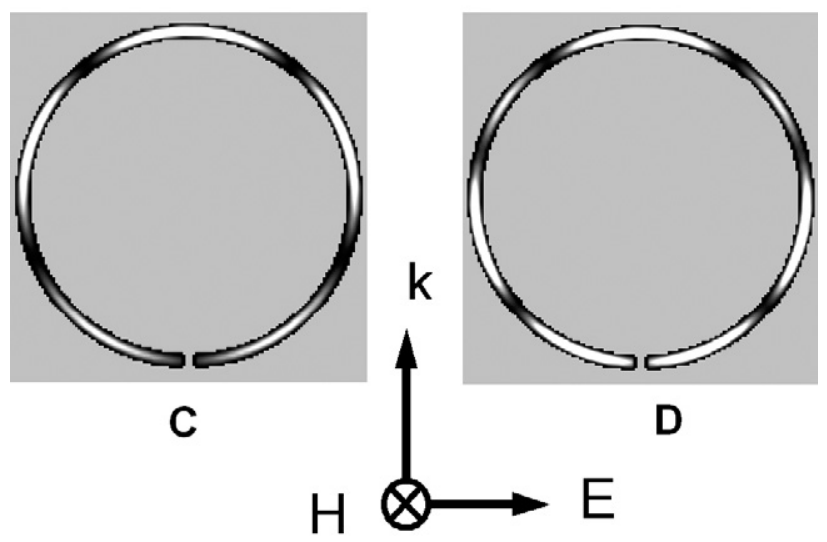

Figure 2. The distribution of the resonant current envelope on the ring at frequencies $\mathrm{A}, \mathrm{B}, \mathrm{C}$ and $\mathrm{D}$ of figure $1(b)$. In the grey scale plot, white represents a large amplitude and black represents zero amplitude. The background colour is irrelevant here; the black contour is the exterior of the ring; the arrow indicates the current direction at a fixed time; the positive and negative signs represent the charges.

stop bands are not only determined by the building blocks but also by the interactions among them.

In order to understand the band gap properties of the ring resonator, the transmission responses of these structures were studied using an approximate analytical formula and the FDTD numerical method. After deducting the gap from the perimeter of a split ring, the resonant frequencies, $f_{n}$, for a separate ring resonator are formulated as [26]

$$
f_{n}=\frac{n c}{(2 \pi r-g) \sqrt{\varepsilon_{\mathrm{eff}}}},
$$

where $n$ is a positive integer, $c$ is the speed of light in vacuum, $r$ is the radius of the Al ring, $g$ is the size of the gap and $\varepsilon_{\text {eff }}$ is the effective dielectric constant. For the square lattice in figure $1, \varepsilon_{\text {eff }}=5.85$ is chosen to fit the first stop band position. The following resonant frequencies are 4.8, 9 and $11.2 \mathrm{GHz}$, which roughly match the measured values of these band gaps. The value of $\varepsilon_{\text {eff }}$ depends on both the structure of the metal elements and the supporting medium in the building block, and the resonant structure usually gives rise to a considerable $\varepsilon_{\text {eff }}$, due to resonance nature, despite the tiny volume fraction of metals. It should be noted that only odd numbers $n$ can satisfy the resonance condition [26].

FDTD is employed here for our simulations due to its reliability in analysing the propagation of EM waves in PBG materials $[27]^{2}$. In the simulations, we first built planar lattices

2 Simulations were performed using the software package CONCERTO 3.1, developed by Vector Fields Limited, England, 2003.

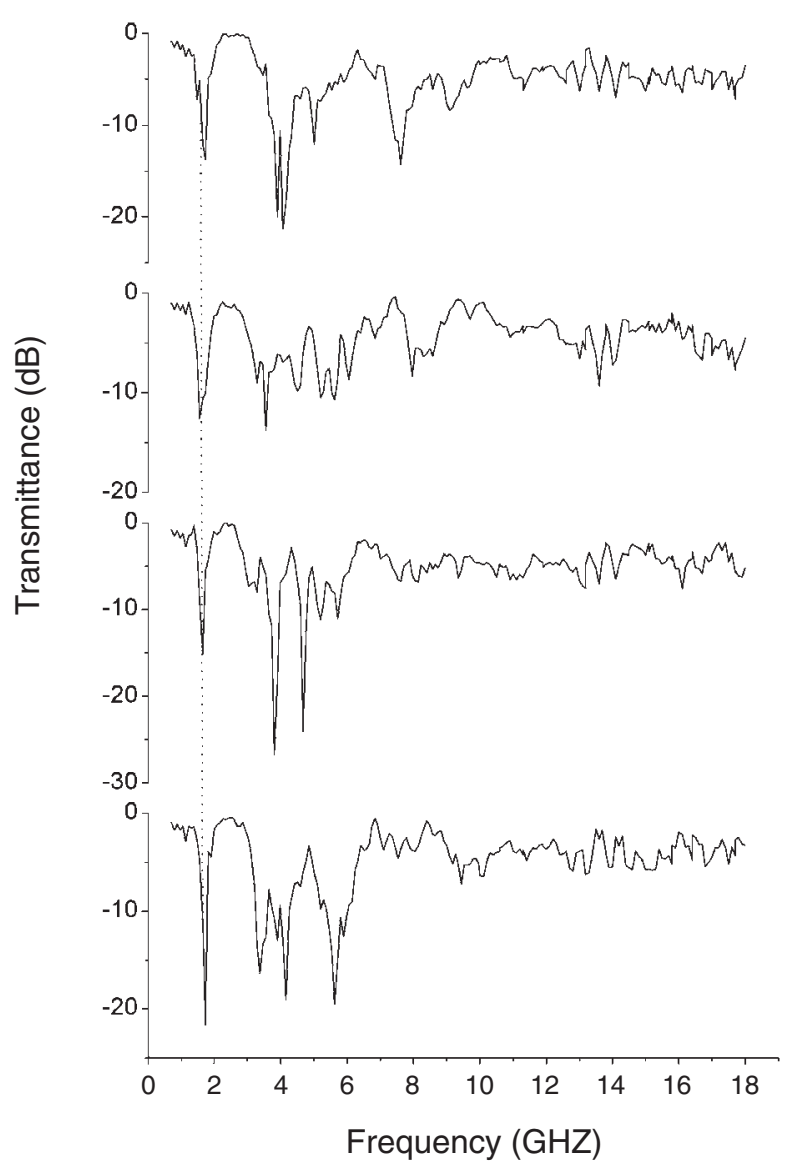

Figure 3. The normal transmittance of the $30 \mathrm{~cm} \times 30 \mathrm{~cm} \times 10 \mathrm{~cm}$ paper box after it is shaken from time to time, where the ring resonators are randomly packed.

consisting of ring resonators and then let a plane wave with a specified polarization direction be incident upon them. For the microwave frequency, we treated aluminium as a perfect metal and extracted the transmission spectrum from the S-parameter calculation. The simulation results, drawn in solid lines in figure 1, can be seen to agree well with the experimental curves. The stop bands A, B, C and D can be understood from the resonant current distributions obtained in the calculations, as illustrated in figure 2. For the principal band $\mathrm{A}$, the current is seen to have the distribution of the first harmonic mode along the circumference of the ring. At both sides of the gap, i.e. two ends of the ring, the charges of the opposite sign accumulate, producing the capacitance for the fundamental mode resonance, while the inductance in the equivalent LC circuit is basically the ring [24]. Because both are formed inside the ring itself, the mode resonance is self-supported, which leads to a robust gap that is determined by the building block and is hardly affected by its neighbours. For the auxiliary bands, the third, fifth and seventh harmonic modes are excited (see figure 2). It can be noted that more and more current nodes appear along the ring. These nodes act like charge reservoirs and store either positive or negative charges during resonances. In figure 2 the oscillating charges and currents at a fixed time are explicitly drawn for the third harmonic mode. The split gap and two nodes divide the ring into three segments where the resonant currents are flowing. Consequently, there 


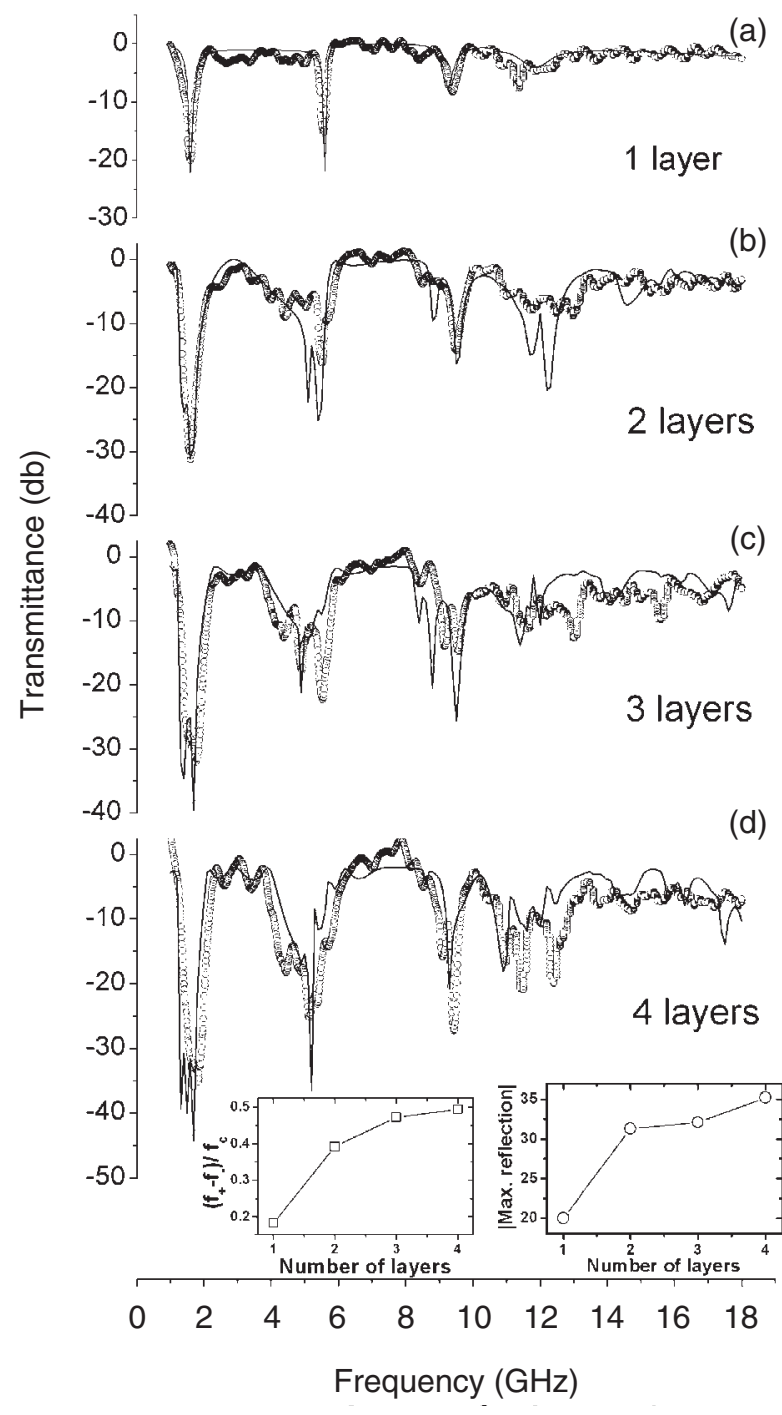

Figure 4. The normal transmittance for a one-layer stack, a two-layer stack, a three-layer stack and a four-layer stack of a square lattice structure. The bandwidth-middle-frequency ratio $\left(\Delta f / f_{c}\right)$ and the maximum rejection are shown in the insets The open circles represent the experimental measurements and the solid lines represent the simulations.

are inter-ring capacitances formed between a ring's node and its neighbours' nodes with opposite charges, in addition to the intra-ring capacitance in the gap. The resonant electric fields concentrated into the capacitances change their distribution accordingly when the structure varies from square lattice to random, since the inter-ring capacitances of a ring are related to its surroundings. Thus, the auxiliary bands shift somewhat with the lattice configuration, and meanwhile their rejections become weak with decreased filling ratio (the area per sphere is $a^{2}$ for a square lattice, $1.3 a^{2}$ for a hexagonal pattern, $1.5 a^{2}$ for a quasicrystal and $2.4 a^{2}$ for random, where $a=28 \mathrm{~mm}$ ), as seen in figure 1. However, the band $\mathrm{A}$, as a fundamental excitation, is expected to survive in any arrangement of building blocks. This can be confirmed by putting the balls in a box $\left(30 \times 30 \times 10 \mathrm{~cm}^{3}\right.$ in volume $)$ in which all the balls are randomly packed and measuring the transmittance of EM waves through the box after shaking it from time to time. The measured results in figure 3 show that the band gap appearing at

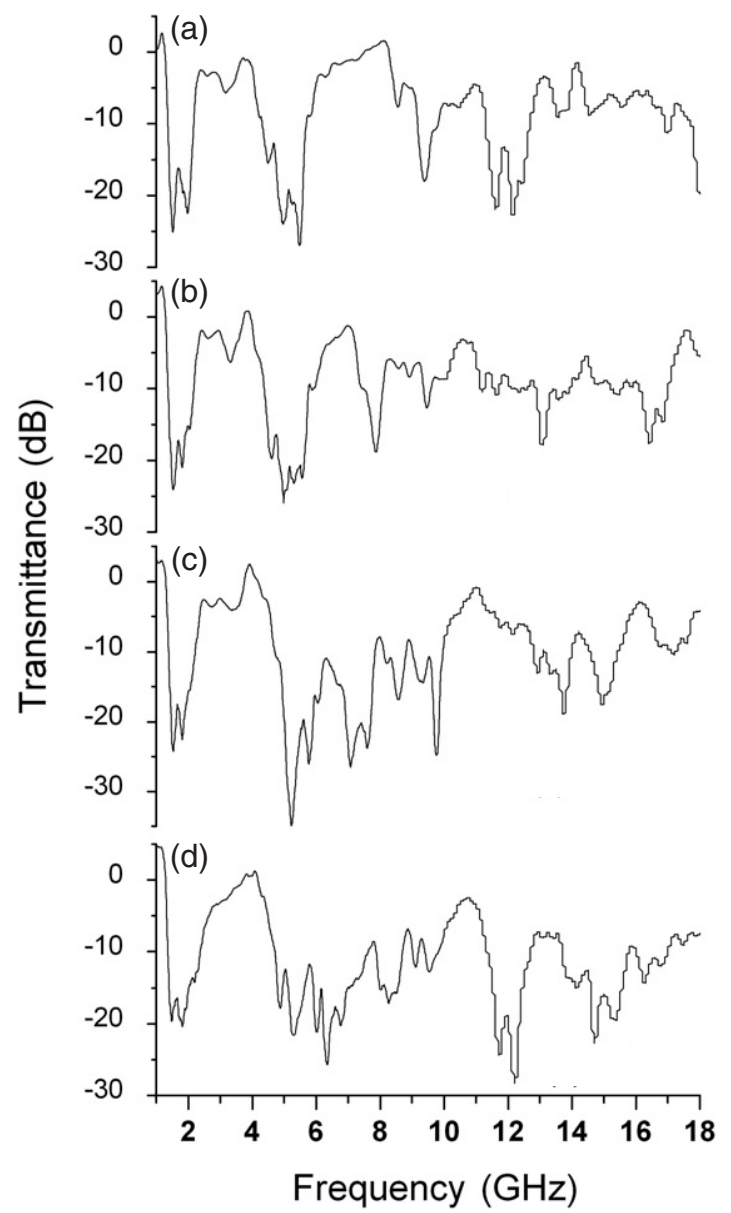

Figure 5. The transmittance measured for a four-layer stack of square lattice structures at incident angles of $(a) 0^{\circ},(b) 15^{\circ},(c) 30^{\circ}$ and $(d) 45^{\circ}$.

a low frequency $(\sim 1.7 \mathrm{GHz})$ remains unchanged which is just the lowest resonant mode. At higher frequencies, the band gaps from the high-order resonances exhibit complex structures, for instance the enormous twin rejection bands at $\sim 4$ and $\sim 5 \mathrm{GHz}$ in the third curves. This is understandable, taking into account the fact that the balls in the box are completely randomized and the resultant transmittance is the hybridization of several effects: multilayer, off-normal incidence and orientations of the ring, which are discussed as follows.

The normal transmission of one- to four-layer stacks of square lattice structures is depicted in figure 4 , where the open circles and solid lines denote the experimental and simulation results, respectively. It can be seen that both the width and the maximum rejection increased when more layers of the same structure were packed together to form a stack. The bandwidth-middle-frequency ratio $\left(\Delta f / f_{c}\right)$ and the maximum rejection are shown in the insets of figure 4 . (The stop band edge is set as the frequency where the transmittance is $-10 \mathrm{~dB}$.) All these are the simple consequence of piling multiple PBG layers together. However, the feature of the individual gap evolving into a serration with increasing layers might be attributed to the coupling of EM fields between layers in both the in-phase and out-of-phase senses [22]. The transmission measured at off-normal incidence is shown in figure 5 for a four-layer stack of square lattices, where the principal stop 


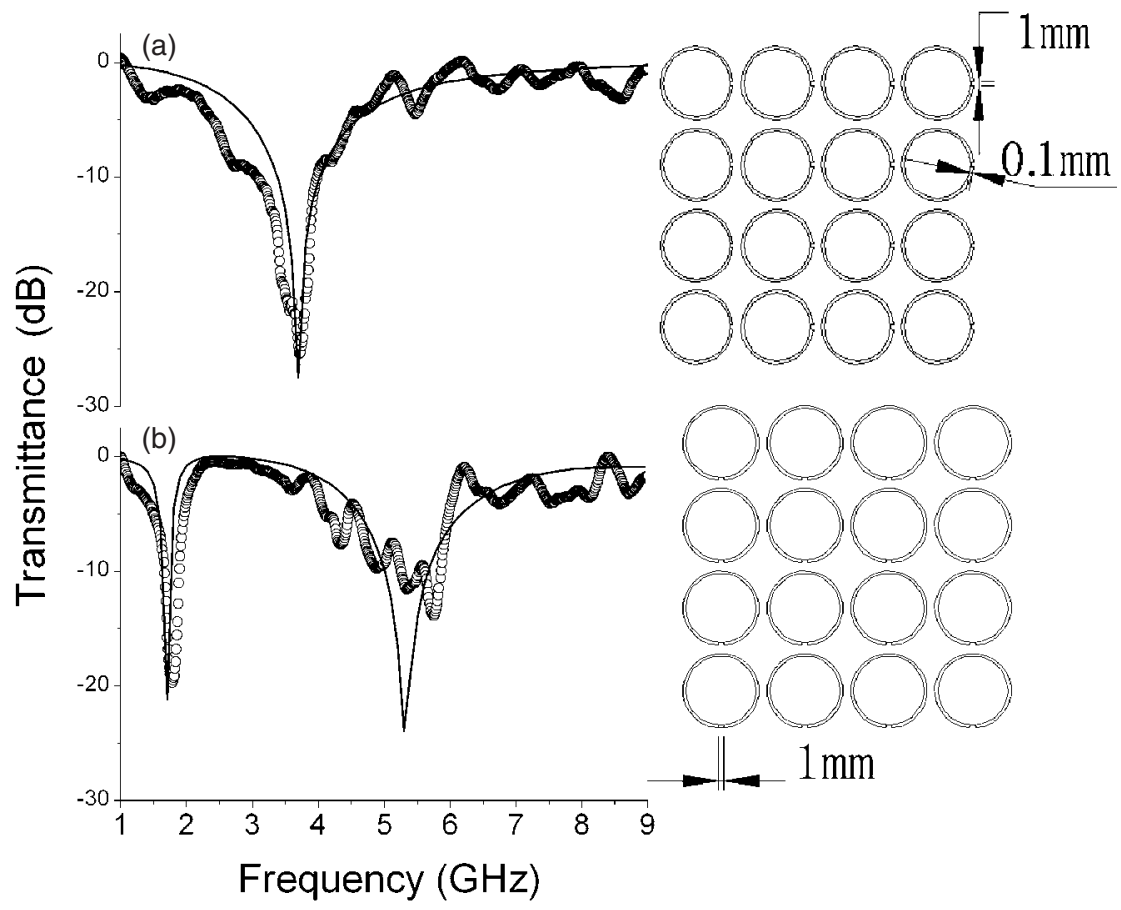

Figure 6. The measured $(\mathrm{O})$ and calculated $(-)$ transmittance at normal incidence of TEM waves for a square lattice structure, into which the resonators are arranged in a way that the ring plane is parallel to the paper substrate with the gap pointing $(a)$ right and $(b)$ down. $E$ is polarized along the horizontal axis and $H$ along the vertical axis.

band is almost static, while the auxiliary bands undergo a frequency shift with the incident angle.

Figure 6 shows the transmittance of the square array of ring resonators for another orientation. Instead of being aligned perpendicular to the paper substrate, the ring plane was arranged parallel to the paper substrate, with the gap openings pointing right and pointing down. Both were arranged in a square lattice structure, and the plane wave was normally incident on them with the $E$ vector polarized in the horizontal direction. Under the illumination condition, no magnetic coupling resonance can be excited, as the incident magnetic field is in-plane with the ring. For the case of the gap pointing to the right, as shown in figure 6(a), one stop band is identified at $3.7 \mathrm{GHz}$ with rejection $-15 \mathrm{~dB}$, while two bands can be detected at $1.8 \mathrm{GHz}$ and $5 \mathrm{GHz}$ with maximum rejection $-20 \mathrm{~dB}$ and $-14 \mathrm{~dB}$, respectively, if the gap is directed downwards (figure $6(b)$ ). The simulation reveals the resonant current distribution of these three bands. The $1.8 \mathrm{GHz}$ and $5 \mathrm{GHz}$ bands in figure $6(b)$, which are found to be the first and third harmonic excitations, have the same current map as the A and B bands in figure 1(b) and, consequently, the band gap positions are consistent with bands $\mathrm{A}$ and $\mathrm{B}$. The consistency of the auxiliary bands in figure $1(b)$ and figure $6(b)$ implies that high-order modes should be electric coupling resonances. The band appearing at $3.7 \mathrm{GHz}$, shown in figure $6(a)$, when the ring resonator has mirror symmetry with respect to the incident $E$ field is proved to be a second harmonic resonance with one node lying at the arc opposite to the gap. We may think that such a resonant frequency is almost two times the fundamental one. It is worth noting that the high-order modes of the ring resonator are induced by the normal incident TEM mode, i.e. zero-order EM waves, and it is not necessary to require an oblique angle of incidence in order to excite them [24].

\section{Conclusion}

We measured the transmission spectra of a single SRR under various types of arrays and different orientations with TEM wave illuminating. We found that the band gaps that originate from different resonant modes localized in the ring have a distinct response to the array type of ring resonators. We discovered that the lowest resonance, self-supported by the intra-ring capacitance, gives rise to the principal stop band, which depends only on the individual building blocks rather than on the block arrangement. It has been pointed out that the negative $\mu$ comes from this resonant mode, and our study here shows the mode is very stable to structural periodicity. However, the remaining auxiliary bands, induced by highorder resonances and appearing in the higher frequency regime, are more sensitive to the configuration of the block array. The bands are also found to be broadened and further attenuated when more layers of identical planar structures are piled up.

\section{Acknowledgment}

We acknowledge support from an RGC Hong Kong research grant through projects 603603 and CA02/03.SC01.

\section{References}

[1] Yablonovitch E 1987 Phys. Rev. Lett. 582486

[2] Fink Y, Winn J N, Fan S, Chen C, Michel J, Joannopoulos J D and Thomas E L 1998 Science 2821679 
[3] Ellis T J and Rebeiz G M 1996 IEEE MTT-S Int. Microwave Symp. Dig. 1157

[4] Radisic V, Qian Y and Itoh T 1998 IEEE Microwave Guid. Wave Lett. 813

[5] Zhang W Y, Lei X Y, Wang Z L, Zheng D G, Tam W Y, Chan C T and Sheng P 2000 Phys. Rev. Lett. 842853

[6] McCalmont J S, Sigalas M M, Tuttle G, Ho K M and Soukolis C M 1996 Appl. Phys. Lett. 682759

[7] Pendry J B 1996 J. Phys.: Condens. Matter 81085

[8] Wang X H, Gu B Y, Li Z Y and Yang G Z 1999 Phys. Rev. B 6011417

[9] Anderson C M and Giapis K P 1996 Phys. Rev. Lett. 77 2949

[10] Wijnhoven J E G and Vos W L 1998 Science 281802

[11] Lopez C, Vazquez L, Meseguer F, Mayoral R, Ocana M and Mguez H 1997 Superlatt. Microstruct. 22399

[12] Toader O and John S 2001 Science 2921133

[13] Brown E R and McMahon O B 1995 Appl. Phys. Lett. 672138

[14] Kao A, McIntosh K A, MaMahon O B, Atkins R and Verghese S 1998 Appl. Phys. Lett. 73145

[15] Serpengüzel A 2002 IEEE Microwave and Wireless Components Lett. 12134
[16] Smith D R, Vier D C, Padilla W, Nemat-Nasser S C and Schultz S 1999 Appl. Phys. Lett. 751425

[17] Pendry J B, Holden A J, Robbins D J and Stewart W J 1999 IEEE Trans. Microwave Theory Tech. 472075

[18] Smith D R, Padilla W, Vier D C, Nemat-Nasser S C and Schultz S 2000 Phys. Rev. Lett. 844184

[19] Shelby R A, Smith D R and Schultz S 2001 Science 29277

[20] Chan Y S, Chan C T and Liu Z Y 1998 Phys. Rev. Lett. 80956

[21] Bayindir M, Cubukcu E, Bulu I and Ozbay E 2001 Phys. Rev. B 63161104

[22] Gay-Balmaz P and Martin O J 2002 J. Appl. Phys. 922929

[23] Katsarakis N, Koschny T, Kafesaki M, Economou E N and Soukoulis C M 2004 Appl. Phys. Lett. 842943

[24] Munk B A 2000 Frequency Selective Surfaces, Theory and Design (New York: Wiley)

[25] Yannopapas V, Modinos A and Stefanou N 1999 Phys. Rev. B 605359

Modinos A, Yannopapas V and Stefanou N 2000 Phys. Rev. B 618099

[26] Kee C S, Jang M Y, Park I, Lim H, Kim J E, Park H Y and Lee J I 2002 Appl. Phys. Lett. 801520

[27] Yee K S 1966 IEEE Trans. Antennas Propag. 14302 